PROCEEDINGS OF THE

AMERICAN MATHEMATICAL SOCIETY

Volume 33, Number 2, June 1972

\title{
MAINTENANCE OF OSCILLATIONS UNDER THE EFFECT OF A PERIODIC FORCING TERM
}

\author{
ATHANASSIOS G. KARTSATOS
}

ABSTRACT. A necessary and sufficient condition is given for the oscillation of all solutions of the differential equation

$$
x^{(n)}+P\left(t, x, x^{\prime}, \cdots, x^{(n-1)}\right)=Q(t)
$$

where $x_{1} P\left(t, x_{1}, x_{2}, \cdots, x_{n}\right)>0$ for every $x_{1} \neq 0$, and $Q$ is a continuous periodic function. This result answers a question recently raised by $\mathrm{J}$. S. W. Wong. It is also shown that a well-known sufficient condition for the existence of at least one nonoscillatory solution of the unperturbed equation guarantees, for a large class of equations, the nonexistence of bounded oscillatory solutions.

Introduction. It is of great importance in physics, and particularly in the study of mechanical systems, to know whether we can maintain the oscillation of all solutions of

$$
x^{(n)}+P\left(t, x, x^{\prime}, \cdots, x^{(n-1)}\right)=0
$$

by adding a periodic forcing term (cf. Wong [5, p. 230], for a question raised there).

In [3] we gave some results concerning the oscillation of solutions to equations of the form

$$
x^{(n)}+P\left(t, x, x^{\prime}, \cdots, x^{(n-1)}\right)=Q(t)
$$

where the function $Q$ was small in some sense. Here we consider the same problem for a class of functions $Q$ which contains "many" continuous periodic functions.

The functions $P, Q$ will be supposed to be continuous and smooth enough to allow the existence of solutions of $(* *)$ for all large $t$. We consider only such solutions in this paper and denote their family by $\mathscr{E}$. A solution $x \in \mathscr{E}$ is said to be bounded if $|x(t)| \leqq k$ for every $t$ in its domain $\left[T_{x},+\infty\right)\left(T_{x} \geqq t_{0}\right.$, where $t_{0}$ is a fixed nonnegative number) and some $k>0$. A solution $x \in \mathscr{E}$ is said to be oscillatory if it has an unbounded set

Received by the editors August 20, 1971 and, in revised form, November 29, 1971. AMS 1969 subject classifications. Primary 3442; Secondary 3440.

Key words and phrases. Oscillation of solutions, nonoscillation of solutions, nonlinear differential equations.

(c) American Mathematical Society 1972 
of zeros on $\left[T_{x},+\infty\right)$. The function $Q$ will be supposed to have the following property: there exist two sequences $\left\{t_{n}\right\},\left\{t_{n}^{*}\right\}$, such that $\lim _{n \rightarrow \infty} t_{n}=$ $\lim _{n \rightarrow \infty} t_{n}^{*}=+\infty, R\left(t_{n}\right)=\lambda_{1}, R\left(t_{n}^{*}\right)=-\lambda_{2}$, and $-\lambda_{2} \leqq R(t) \leqq \lambda_{1}$ for all $t \in\left[t_{0},+\infty\right)$, where $R \in C^{n}\left[t_{0},+\infty\right), R^{(n)}(t)=Q(t)$ for every $t \in\left[t_{0},+\infty\right)$, and $\lambda_{1}, \lambda_{2}>0$.

1. Our main result is contained in the following

THEOREM 1. Suppose that $P\left(t, x_{1}, x_{2}, \cdots, x_{n}\right) \equiv P_{0}(t) G\left(x_{1}, x_{2}, \cdots, x_{n}\right)$ where

(i) $P_{0}:\left[t_{0},+\infty\right) \rightarrow(0,+\infty)$, continuous and such that for any continuous $S:\left[t_{0},+\infty\right) \rightarrow(0,+\infty)$ with $S(t) \geqq P_{0}(t), t \in\left[t_{0},+\infty\right)$, the equation

$$
W^{(n)}+S(t) G\left(W, W^{\prime}+R^{\prime}, \cdots, W^{(n-1)}+R^{(n-1)}\right)=0
$$

has all of its bounded solutions (resp. all of its solutions) (a) for $n=o d d$, oscillatory or tending monotonically to zero, (b) for $n=e v e n$, oscillatory;

(ii) $G: R^{n} \rightarrow R=(-\infty,+\infty)$, continuous, increasing w.r.t. $x_{1}$ and $x_{1} G\left(x_{1}, x_{2}, \cdots, x_{n}\right)>0$ for every $x_{1} \neq 0$.

Then, all bounded solutions (resp. all solutions) $x \in \mathscr{E}$ are (a) for $n=o d d$, oscillatory or such that $\lim _{t \rightarrow+\infty}[x(t)-R(t)]=-\lambda_{1}$ or $\lambda_{2}$, (b) for $n=e v e n$, oscillatory.

Proof. Suppose first that $n=$ even and that (1) has all of its bounded solutions oscillatory. Assume the existence of a bounded nonoscillatory solution $x \in \mathscr{E}$ and let $0<x(t) \leqq k<+\infty$ for all $t \geqq t_{1}$, where $t_{1} \geqq t_{0}$. Then the function $W(t) \equiv x(t)-R(t)$ is a bounded solution of

$$
W^{(n)}+P_{0}(t) G\left(W(t)+R(t), \cdots, W^{(n-1)}(t)+R^{(n-1)}(t)\right)=0
$$

with the property $W(t)+R(t)>0$ on $\left[t_{1},+\infty\right)$. For this $W(t)$ we obtain $W^{(n)}(t)<0$, i.e., as in Theorem 1 of $[1],(-1)^{m} W^{m}(t)<0$ for every $m=$ $1,2, \cdots, n$ and every $t \in\left[t_{1},+\infty\right)$. Since $W(t)+R(t)>0, W^{\prime}(t)>0$ and $R\left(t_{n}^{*}\right)=-\lambda_{2}$, there exists $n_{0}$ such that $t_{n_{0}}^{*} \geqq t_{1}$ and $W(t)+R(t) \geqq W\left(t_{n_{0}}^{*}\right)-$ $R\left(t_{n_{0}}^{*}\right)=W\left(t_{n_{0}}^{*}\right)-\lambda_{2}>0, t \in\left[t_{n_{0}}^{*},+\infty\right)$. Thus,

$$
\begin{aligned}
G(W+R, & \left.W^{\prime}+R^{\prime}, \cdots, W^{(n-1)}+R^{(n-1)}\right) \\
& \geqq G\left(W-\lambda_{2}, \cdots, W^{(n-1)}+R^{(n-1)}\right)>0 \text { for } t \in\left[t_{n_{0}}^{*},+\infty\right) .
\end{aligned}
$$

Now put $V(t) \equiv W(t)-\lambda_{2}, t \in\left[t_{n_{0}}^{*},+\infty\right)$; then (1) becomes

(3) $V^{(n)}(t)+S(t) G\left(V(t), V^{\prime}(t)+R^{\prime}(t), \cdots, V^{(n-1)}(t)+R^{(n-1)}(t)\right)=0$

where

$$
S(t)=\frac{P_{0}(t) G\left(W+R, \cdots, W^{(n-1)}+R^{(n-1)}\right)}{G\left(W-\lambda_{2}, \cdots, W^{(n-1)}+R^{(n-1)}\right)} \geqq P_{0}(t) .
$$


Since $V(t)$ has to be oscillatory by assumption, we obtain a contradiction to $W(t)-i_{2}>0$. Consequently, $x(t)$ cannot be eventually positive. Av analogous proof holds if we assume that $x(t)$ is negative for all large $t$, and the proof for bounded solutions is complete.

Now, suppose that $x(t)$ is a nonoscillatory and unbounded solution of (**). Assume that $x(t)>0$ for $t \geqq t_{1}$. Then the function $W(t) \equiv x(t)-R(t)$ satisfies equation (2) and, according to Theorem 2 of [1], we must have $W^{\prime \prime}(t)>0$ for all large $t$ and $\lim _{t \rightarrow+\infty} W(t)=+\infty$. Thus, $W(t)+R(t) \geqq$ $W(t)-\lambda_{2}>0$ eventually, and we arrive again at equation (3) which (provided of course that hypothesis (i), (b) is satisfied) implies a contradiction to the positivity of the function $W(t)-\lambda_{2}$. Consequently, $x(t)$ cannot be eventually positive. An analogous situation appears in the case $x(t)=$ negative for all large $t$, and this completes the proof in the case $n=$ even.

Assume now that $n=$ odd, $x(t)$ is a solution of $(*)$ such that $0<x(t) \leqq k$, $t \in\left[t_{3},+\infty\right)$ and hypothesis (i), (a) is satisfied for the bounded solutions of (1). Let $W(t) \equiv x(t)+R(t), t \in\left[t_{1},+\infty\right)$. Then $W(t)$ is a bounded solution of (2) such that $W(t)+R(t)>0$ and $W^{\prime}(t)<0$ for every $t \in\left[t_{1},+\infty\right)$ (formulas analogous to those of Cases I, II in Theorem 2 of [1] also hold in the case $n=0 \mathrm{dd})$. Suppose that $W(\tau)-\lambda_{2} \leqq 0$ for scme $\tau \geqq t_{1}$. Then, $W(t)-\lambda_{2}<0$ for all $t>\tau$, which implies a contradiction to $W(t)+R(t)>0$. Thus, $W(t)-\lambda_{2}>0$ for all $t \geqq t_{1}$ and it follows frem (3) that $\lim _{t \rightarrow+\infty} W(t)-$ $\lambda_{2}=0$, or $\lim _{t \rightarrow+\infty} x(t)-R(t)=\lambda_{2}$. As in the case $n=$ even, it can ice shown that there are no positive solutions of $(* *)$ which are unbounded for all large $t$, and this completes the proof of the case $n=$ odd.

COROLlaRY. If hypothesis (ii) of Theorem 1 is satisfied, and $P_{0}$ is positive and continuous with $\int_{i_{0}}^{\infty} t^{n-1} P_{0}(t) d t=+\infty$, then, for $n=e v e n$, every bounded solution of $(* *)$ is oscillatory, and, for $n=o d d$, every bounded solution of $(* *)$ is osciliatory, or such that $\lim _{t \rightarrow+\infty}[x(t)-R(t)]=-\lambda_{1}$ or $\lambda_{2}$.

2. Let $x(t)$ he a solution of $(* *)(Q(t) \equiv 0)$ such that $|x(t)| \leqq k, t \in$ $\left[T_{x},+\infty\right)$ and

$$
\int_{T_{x}}^{\infty} t^{n-1}\left|P\left(t, x(t), x^{\prime}(t), \cdots, x^{(n-1)}(t)\right)\right| d t<+\infty .
$$

Then we have

$$
x^{(n-1)}(t)=x^{(n-1)}\left(T_{x}\right)-\int_{\Gamma_{x}}^{t} P(s, \bar{x}(s)) d s \quad\left(\bar{x}(t) \equiv\left(x(t), x^{\prime}(t), \cdots, x^{(n-1)}(t)\right)\right)
$$

which, by use of (4), yields

$$
\lim _{t \rightarrow+\infty} x^{(n-1)}(t)=x^{(n-1)}\left(T_{x}\right)-\int_{T_{x}}^{\infty} P(t, \bar{x}(t)) d t .
$$


Now, $\lim _{t \rightarrow+\infty} x^{(n-1)}(t)=0$, otherwise we would have $\lim _{t \rightarrow+\infty} x(t)=$ $\pm \infty$, a contradiction to the boundedness of $x(t)$. Thus, from (5) we obtain

$$
x^{(n-1)}\left(T_{x}\right)=\int_{T_{x}}^{\infty} P(t, \bar{x}(t)) d t .
$$

It is obvious that we can replace $\left(T_{x}\right)$ in (6) by any $t \geqq T_{x}$, in which case it becomes

$$
x^{(n-1)}(t)=\int_{t}^{\infty} P(s, \bar{x}(s)) d t, \quad t \geqq T_{x} .
$$

A new integration from $T_{x}$ to $t \geqq T_{x}$ gives

$$
\begin{aligned}
x^{(n-2)}(t) & =x^{(n-2)}\left(T_{x}\right)+\int_{T_{x}}^{t}\left(\int_{s}^{\infty} P(u, \bar{x}(u)) d u\right) d s \\
= & x^{(n-2)}\left(T_{x}\right)+\int_{T_{x}}^{\infty}\left(s-T_{x}\right) P(s, \bar{x}(s)) d s-\int_{t}^{\infty}(s-t) P(s, \bar{x}(s)) d s .
\end{aligned}
$$

Taking the limit of (8) as $t \rightarrow+\infty$ and then replacing $T_{x}$ by $t \geqq T_{x}$, we finally obtain

$$
x^{(n-2)}(t)=\int_{t}^{\infty}(t-s) P(s, \bar{x}(s)) d s
$$

Repeating the same process we obtain the formulas

$$
x^{(m)}(t)=\int_{t}^{\infty} \frac{(t-s)^{n-m-1}}{(n-m-1) !} P(s, \bar{x}(s)) d s, \quad m=1,2, \cdots, n-1,
$$

and

$$
\begin{aligned}
x(t)= & x\left(T_{x}\right)-\int_{T_{x}}^{\infty} \frac{\left(T_{x}-s\right)^{n-1}}{(n-1) !} P(s, \bar{x}(s)) d s \\
& +\int_{t}^{\infty} \frac{(t-s)^{n-1}}{(n-1) !} P(s, \bar{x}(s)) d s .
\end{aligned}
$$

If $x(t)$ is bounded and oscillatory, then we have to have $\lim _{t \rightarrow+\infty} x(t)=0$. Thus, from (11) we get

$$
x(t)=\int_{t}^{\infty} \frac{(t-s)^{n-1}}{(n-1) !} P(s, \bar{x}(s)) d s .
$$

We have the following

LEMMA. If $x(t)$ is a solution of $(* *)(Q(t) \equiv 0)$ such that $|x(t)| \leqq k$, $t \in\left[T_{x},+\infty\right)$ and

$$
\int_{T_{x}}^{\infty} t^{n-1}|P(t, \bar{x}(t))| d t<+\infty
$$

then $x(t)$ satisfies the equation (11), which reduces to (12) if $x(t)$ is oscillatory. 
It has been repeatedly shown (see Wong [5] and the references cited there) that if $\int_{t_{0}}^{\infty} t^{n-1}\left|P_{0}(t)\right| d t<+\infty$, the equation (**) with $Q(t) \equiv 0$ and $P \equiv P_{0} G$ has at least one bounded nonoscillatory solution which converges to a nonzers limit as $t \rightarrow+\infty$. We show here that, under quite general conditions, equation $(* *)$ with $Q(t) \equiv 0$ has no bounded oscillatory solutions. Before we give the main theorem of this section, we note that the integral condition on $P_{0}$ in the corollary is also necessary. In fact, as above, the problem reduces tc finding a solution to the integral equation

$x(t)=k+\int_{t}^{\infty} \frac{(t-s)^{n-1}}{(n-1) !} P_{0}(s) G\left(W(s)+R(s), \cdots, W^{(n-1)}(s)+R^{(n-1)}(s)\right) d s$,

where $k$ is a nonzero constant.

THEOREM 2. Let $Q(t) \equiv 0$ in $(* *)$ and

(i) $P:\left[t_{0},+\infty\right) \times R^{n} \rightarrow R$, continuous, and such that

$$
\begin{aligned}
\left|P\left(t, u(t), u^{\prime}(t), \cdots, u^{(n-1)}(t)\right)\right| & \leqq P_{1, u}(t)|u(t)|, \\
\int_{t_{0}}^{\infty} t^{n-1} P_{1, u}(t) d t & <+\infty,
\end{aligned}
$$

for every bounded $u \in C^{n}\left[t_{0},+\infty\right)$, where $P_{1, u}:\left[t_{0},+\infty\right) \rightarrow R_{+}=[0,+\infty)$ and continuous.

Then, every bounded oscillatory $x \in \mathscr{E}$ has to be idertically zero for all large $t$.

Proof. Suppose that $x \in \mathscr{E}$ is bounded and oscillatory. Then it follows from the Lemma that $\lim _{t \rightarrow+\infty} x(t)=0$ and

$$
x(t)=\int_{t}^{\infty} \frac{(t-s)^{n-1}}{(n-1) !} P(s, \bar{x}(s)) d s .
$$

Now, there exists $t_{1} \geqq T_{x}$ such that $\left|x\left(t_{1}\right)\right|=\sup _{t \in\left[t_{2},+\infty\right)}|x(t)|$, and

$$
\int_{t_{1}}^{\infty}\left(t-t_{1}+1\right)^{n-1} P_{1 . x}(t) d t<1 .
$$

Combining (13) and (14) we obtain

$$
|x(t)| \leqq \int_{t}^{\infty}\left|(t-s)^{n-1} P(s, x(s))\right| d s \leqq \int_{t}^{\infty}\left(t-t_{\mathrm{i}}+1\right)^{n-1} P_{1, x}(t)|x(t)| d t
$$

and

$$
\left|x\left(t_{1}\right)\right| \leqq\left|x\left(t_{1}\right)\right| \int_{t_{1}}^{\infty}\left(t-t_{1}+1\right)^{n-1} P_{1, x}(t) d t,
$$

a contradiction to (14), unless $\sup _{t \in\left[t_{1},+\infty\right)}|x(t)|=0$. 
A corollary to Theorem 2, which covers large classes of interesting equations, is the following

COROLLARY. There are no nontrivial bounded oscillatory solutions to the equation

$$
x^{(n)}+\left(1 / t^{n+\varepsilon}\right) x^{2 p+1}=0
$$

where $n \geqq 1, p=a$ nonnegative integer, $\varepsilon>0$.

In fact, here we have $P_{\llcorner, x}(t)=k^{2 p}\left(1 / t^{n+\varepsilon}\right)$ for any $x \in C^{n}[1,+\infty)$, $|x(t)| \leqq k$.

Discussion. It is worth noticing that the problem of the oscillation of $(* *)$ is being reduced here to that concerning the oscillation of an equation of the type (*) without forcing term. However, there still remains an open question: What functions do ensure the oscillation of $(* *)$ without necessarily requiring that all solutions of the unperturbed equation be oscillatory?

Theorem 1 can be extended to equations with more general functions $P$, e.g., the ones considered in Chapter 1 of [2] which contain as very special cases some of those considered by Ryder and Wend in [4].

Another open problem here is the following: what happens if $P$ does not satisfy $x_{1} P\left(t, x_{1}, \cdots, x_{n}\right)>0$ ? In the case

$$
P^{-}(t, \bar{x}(t))=-\min \{P(t, \bar{x}(t)), 0\}=\text { small enough, }
$$

e.g., $\int_{t_{0}}^{\infty} t^{n-1} P^{-}(t, \bar{x}(t)) d t<+\infty$, the author thinks that the following procedure might prove to be useful: We first reduce the problem to that of an unperturbed equation and then we consider the perturbed equation

$$
\begin{aligned}
& W^{(n)}+P^{+}\left(t, W+R, \cdots, W^{(n-1)}+R^{(n-1)}\right) \\
& \quad=P^{-}\left(t, W+R, \cdots, W^{(n-1)}+R^{(n-1)}\right) \\
& \quad\left(P^{+}(t, \bar{x}(t))=\max \{P(t, \bar{x}(t)), 0\}\right)
\end{aligned}
$$

which can be treated as in [3].

EXAmPle. Consider the equation:

$$
x^{(n)}+\left(1 / t^{n}\right) x^{2 p+1}=\sin (2 t+1)
$$

where $n=$ even, $p=$ a positive integer. Here we have

Since

$$
\begin{aligned}
P_{0}(t) & \equiv 1 / t^{n}, \quad G\left(x_{1}, x_{2}, \cdots, x_{n}\right) \equiv x_{1}^{2 p+1}, \\
R(t) & \equiv 2^{-n} \sin (2 t+1) .
\end{aligned}
$$

$$
\int_{1}^{\infty} t^{n-1} S(t) d t=+\infty \text { for any function } S(t) \geqq P_{0}(t)
$$

it follows from Theorem 2 in [1] that for such functions $S$ all solutions of 
$x^{(n)}+S(t) x^{2 p+1}=0$ are oscillatory, and our Theorern 1 implies the oscillation of all solutions of $(* * *)$.

The author wishes to thank the referee for his helpful suggestions.

NoTE ADDED IN PROOF. Professor H. Teufel [Forced second order nonlinear oscillation, J. Math. Anal. Appl. (to appear)] has obtained some results under conditions indepencient of the ones considered in this paper.

\section{REFERENCES}

1. A. G. Kartsatos, On oscillation of solutions of even order nonlinear differential equations, J. Differential Equations 6 (1969), 232-237. MR 39 \#587?.

2. Contributions to the research of the oscillation and the asymptotic behaviour of solutions of ordinary differential equations, Doctoral Disserration, University of Athens; Bull. Soc. Math. Grèce 10 (1969), 1-48.

3. - On the maintenance of oscillations of nth order equations under the effect of a smali forcing term, J. Differential Equations 10 (1971), 355-363.

4. G. H. Ryder and D. V. V. Wend, Oscillation of solutions of certain ordinary differential equations of nth order, Proc. Amer. Math. Soc. 25 (1970), 463-469. MR 41 \#5710.

5. J. S. W. Wong, On second order nonlinear oscillation, Funkcial. Ekvac. 11 (1968), 207-234. MR 39 \#7221.

Department of Mathematics, University of SOUth Florida, Tampa, Florida 33620 